\title{
839 MICROBIOTA-SPECIFIC T FOLLICULAR HELPER CELLS DRIVE TERTIARY LYMPHOID STRUCTURE FORMATION AND ANTI-TUMOR IMMUNITY IN COLORECTAL CANCER
}

Abigail Overacre-Delgoffe*, Hannah Bumgarner, Anthony Cillo, Ansen Burr, Justin Tometich, Amrita Bhattacharjee, Tullia Bruno, Dario Vignali, Timothy Hand. University of Pittsburgh, Pittsburgh, PA, USA

Background Colorectal cancer (CRC) is one of the most common and deadly cancers in the US, and the survival rate for advanced cases is poor. While immunotherapy has revolutionized cancer treatment, CRC remains largely unresponsive, with only $\sim 6 \%$ of patients responding to anti-PD1. Specific microbiome signatures are associated with anti-PD1 response in melanoma patients; however, the underlying mechanism remains unclear. While the microbiome in cancer patients has been extensively studied, the endogenous immune response to these microbes and the subsequent effects on cancer immunity remain unstudied. Most microbes reside within the gut, and bacteria that adhere to the intestinal epithelium can stimulate bacteria-specific immune responses. Therefore, we hypothesized that the microbiome, especially adherent, immunogenic bacteria, may support anti-tumor immunity through activation of local microbiota-specific $\mathrm{T}$ cells.

Methods Using a carcinogen-induced mouse model of CRC, we sought to determine the impact of microbiome modulation on the anti-tumor immune response. We colonized tumor-bearing mice with Helicobacter hepaticus (Hhep) and assessed tumor burden, survival, and immune infiltration. Lymphocytes were isolated from the tumor and surrounding tissue when tumors were terminal (12 weeks). We utilized TCR transgenic mice and MHC class II tetramers to track the spatial and transcriptional Hhep-specific $\mathrm{T}$ cell response through 5' single cell RNAseq, flow cytometry, and spectral immunofluorescence.

Results Hhep colonization in tumor-bearing mice led to decreased tumor burden and significantly improved survival. Interestingly, colonization induced activation of Hhep-specific $\mathrm{T}$ follicular helper cells (TFHs) that supported formation of mature peri- or intra-tumoral tertiary lymphoid structures (TLS). The presence of TLS led to increased infiltration of cytotoxic lymphocytes ( $\mathrm{T}$ and NK cells) within the tumor core. Surprisingly, the anti-tumor response was dependent on $\mathrm{CD} 4+\mathrm{T}$ and $\mathrm{B}$ cells but not CD8 $+\mathrm{T}$ cells. Using TFH KO mice, we found that Hhep-specific CD4+ T cells were both necessary and sufficient to drive TLS maturation and antitumor immunity.

Conclusions Here, we demonstrate that addition of a single bacterial species after tumor formation leads to a reduction in CRC tumor burden and increased survival through TLS maturation. This microbiome-dependent remodeling of the tumor microenvironment is driven by Hhep-specific TFH cells that are both necessary and sufficient for tumor control, demonstrating for the first time that microbiota-specific $\mathrm{T}$ cells contribute to anti-tumor immunity. Overall, these findings suggest that microbiome modulation and the subsequent microbiotaspecific CD4+ $\mathrm{T}$ cell response may represent a new variety of immunotherapies for cancers that remain resistant to checkpoint blockade.

http://dx.doi.org/10.1136/jitc-2021-SITC2021.839 